\title{
Fast Assembly of Gold Nanoparticles in Large-Area 2D Nanogrids Using a One-Step, Near-Infrared Radiation-Assisted Evaporation Process
}

André Utgenannt, ${ }^{\dagger}$ Ross Maspero, ${ }^{\dagger, \ddagger}$ Andrea Fortini, ${ }^{\dagger}$ Rebecca Turner, ${ }^{\dagger}$ Marian Florescu, ${ }^{\dagger, \ddagger}$ Christopher Jeynes, ${ }^{\S}$ Antonios G. Kanaras, ${ }^{\|}$Otto L. Muskens, ${ }^{\|}$Richard P. Sear, ${ }^{\dagger}$ and Joseph L. Keddie ${ }^{*}$,

${ }^{\dagger}$ Department of Physics, ${ }^{\ddagger}$ Advanced Technology Institute, and ${ }^{\S}$ Ion Beam Centre, University of Surrey, Guildford, Surrey GU2 7XH, U.K. "Physics and Astronomy, Faculty of Physical Sciences and Engineering, University of Southampton, Highfield, Southampton, SO17 1BJ, U.K.

Supporting Information
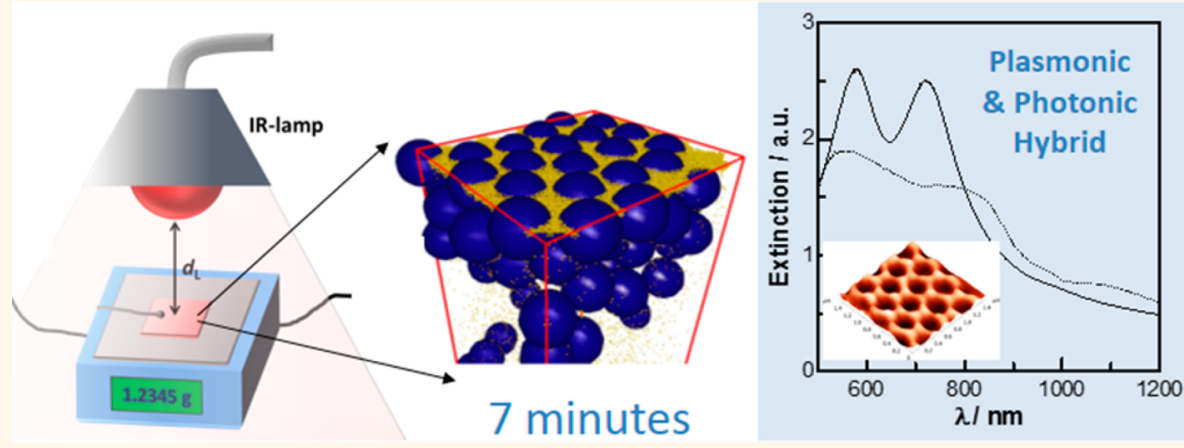

ABSTRACT: When fabricating photonic crystals from suspensions in volatile liquids using the horizontal deposition method, the conventional approach is to evaporate slowly to increase the time for particles to settle in an ordered, periodic closepacked structure. Here, we show that the greatest ordering of $10 \mathrm{~nm}$ aqueous gold nanoparticles (AuNPs) in a template of larger spherical polymer particles (mean diameter of $338 \mathrm{~nm}$ ) is achieved with very fast water evaporation rates obtained with near-infrared radiative heating. Fabrication of arrays over areas of a few $\mathrm{cm}^{2}$ takes only $7 \mathrm{~min}$. The assembly process requires that the evaporation rate is fast relative to the particles' Brownian diffusion. Then a two-dimensional colloidal crystal forms at the falling surface, which acts as a sieve through which the AuNPs pass, according to our Langevin dynamics computer simulations. With sufficiently fast evaporation rates, we create a hybrid structure consisting of a two-dimensional AuNP nanoarray (or "nanogrid") on top of a three-dimensional polymer opal. The process is simple, fast, and one-step. The interplay between the optical response of the plasmonic Au nanoarray and the microstructuring of the photonic opal results in unusual optical spectra with two extinction peaks, which are analyzed via finite-difference time-domain method simulations. Comparison between experimental and modeling results reveals a strong interplay of plasmonic modes and collective photonic effects, including the formation of a high-order stopband and slow-light-enhanced plasmonic absorption. The structures, and hence their optical signatures, are tuned by adjusting the evaporation rate via the infrared power density.

KEYWORDS: self-assembly, plasmonics, photonics, coupling, gold nanoparticles, inverse opal, evaporation, IR heating

$\mathrm{P}$ eriodic nanostructures have a wide range of applications including antireflection coatings, ${ }^{1}$ self-cleaning films, ${ }^{2}$ sensors, ${ }^{3,4}$ metamaterials, ${ }^{5}$ and devices for energy harvesting. ${ }^{6}$ Among the methods for the fabrication of periodic nanostructures, self-assembly methods are particularly attractive because of their numerous benefits, including low cost and simple processing steps. ${ }^{7}$ Vertical $^{8}$ and horizontal ${ }^{9}$ deposition of nanoparticles from a carrier solvent are established methods for the fabrication of periodic nanostructures.
For both types of deposition method, several groups have investigated how the quality of an ordered nanoparticle assembly is affected by various parameters, e.g., the evaporation rate (controlled via humidity, ${ }^{10}$ air pressure, ${ }^{11}$ solvent volatility, ${ }^{12}$ etc.), the withdrawal speed of the plate in confined geometries, ${ }^{13}$ the

Received: November 1, 2015

Accepted: January 14, 2016 
particle concentration, and the particle size ratio in bimodal blends. ${ }^{14}$ Horizontal deposition is considered to be a low-cost and fast method with the ability to be employed in the large-scale fabrication of colloidal crystals. ${ }^{15}$ Its main advantage lies in its ease of use and relatively low technical difficulty compared to vertical deposition methods. The environmental conditions (for example, the temperature, pressure, and humidity) during the drying process have a pronounced influence on the structure obtained via the horizontal deposition method. However, according to previous reports, the method typically suffers from long drying times, which are conventionally used in colloidal crystallization from liquids to obtain a high degree of periodicity and order. ${ }^{16}$ This time inefficiency presents a major obstacle to the widespread adoption of the horizontal deposition method.

The aim of most published work on particle assembly is to create either 2D structures (colloidal monolayers, nanomeshes, ${ }^{17,18}$ ring structures, ${ }^{4}$ and arrays of nanocavities ${ }^{19}$ ) or 3D structures (opals and inverse opals). In recent years, hybrid colloidal crystals, which consist of 2D plasmonic arrays on photonic crystals, ${ }^{20}$ are of growing interest. Hybrid structures combine aspects of plasmonics and photonics. ${ }^{21}$ The strong synergetic effect of plasmon excitation and the photonic band gap results in materials with advanced optical functionalities that enable the control of the propagation, emission, and extinction of light on the nanoscale. These materials represent a promising platform for novel light sources and sensors. ${ }^{20}$ The broad variety of designs for $2 \mathrm{D}$ nanoarrays and 3D structures results in a vast number of possible hybrid architectures. Hence, a rapid fabrication method will be highly beneficial to progress in this field.

Here, we introduce a fast, efficient, and low-cost fabrication method to achieve the colloidal self-assembly of a hybrid structure consisting of a (quasi) two-dimensional (2D) gold nanoparticle network (which we call a "nanogrid") and a threedimensional binary colloidal crystal (3D bCC). Surprisingly, a highly ordered periodic nanostructure is obtained only with a high evaporation rate induced with infrared radiative heating. In our previous work, we spatially modulated the infrared power density across the surface of a wet colloidal film. ${ }^{22}$ We showed that nanoparticles could be transported laterally in the plane of the film to create dome-like surfaces in a periodic array. In the present work, we have discovered that the fast evaporation rates obtained with infrared heating drive the assembly of nanoparticles at the descending film surface.

We show how the ordering of the nanostructure benefits from shorter fabrication times, which contradicts the commonly held belief that slow drying with long assembly times is needed to obtain structures with a high degree of periodicity. Additionally, we show that the surface morphology of the hybrid structures obtained can be adjusted simply by changes in the evaporation rate without the need for a sophisticated experimental setup or additional processing steps.

The combination of the controlled materials fabrication with advanced structural characterization and optical experiments allows us to relate the specific structure of these new hybrid plasmonic-photonic crystals with their optical properties. An intricate interplay between plasmonic resonances and photonic band structure effects is found, which shows the promise of fast evaporative techniques in achieving new types of hybrid optical materials.

\section{RESULTS AND DISCUSSION}

Fabrication, Optical Properties, and Structure of Colloidal Nanogrids. Stabilized gold nanoparticles (AuNPs), approximately $10 \mathrm{~nm}$ diameter, in water were blended with spherical acrylic copolymer particles $(338 \mathrm{~nm})$ in water. ${ }^{22}$ The dispersions were cast as films and dried over a range of evaporation rates, which are expressed as the velocity at which the water surface falls downward during drying and which were determined experimentally.

Figure 1a shows UV-vis-NIR spectra from a series of dried thick films of AuNP/polymer particle blends fabricated using different evaporation rates, $\dot{E}$, in descending order between $2.08 \times 10^{-6}$ and $0.36 \times 10^{-6} \mathrm{~m} \mathrm{~s}^{-1}$. This range of $\dot{E}$ was achieved by using near-infrared (NIR) radiative heating with a corresponding range of power densities. The spectra obtained from films dried normally under ambient conditions $(\dot{E}=1.1 \times$ $\left.10^{-7} \mathrm{~ms}^{-1}\right)$ and slowly dried under high humidity $(\dot{E}=3.2 \times$ $10^{-9} \mathrm{~ms}^{-1}$ ) are included for comparison. The spectrum of the fastest dried film shows two pronounced peaks with maxima at $\lambda_{\text {max }, \mathrm{I}}=574 \mathrm{~nm}$ and $\lambda_{\text {max }, \mathrm{II}}=716 \mathrm{~nm}$. The intensities of the peaks weaken when the evaporation rate is slowed, even though the gold concentration in all films is identical. Moreover, the assembly time increases from only $7 \mathrm{~min}$ for the fastest evaporation rate to more than $2 \mathrm{~h}$ under ambient conditions.

Notably, the spectra for the normally and slowly dried films show only a broad extinction across the whole range of measured wavelengths without any specific spectral features as a signature of a periodic structure. Periodically structured materials show a specific optical response, for example, through extinction over a certain wavelength range. Variations in the structure cause changes in the spectrum, which are visible through shifts of peaks and an increase or decrease in the signal intensity. ${ }^{23,24}$ The development of the spectra in Figure 1a strongly indicates that a highly periodic structure can be fabricated on a short time scale through rapid drying in a horizontal deposition process.

The SEM images in Figure $1 b-d$ show the surface structure that was fabricated using the fastest evaporation rate $(\dot{E}=2.08 \times$ $\left.10^{-6} \mathrm{~m} \mathrm{~s}^{-1}\right)$. In these images, the polymer particles appear dark, and the AuNPs appear white. The polymer particles are arranged in ordered hexagonal arrays at the surface, and the AuNPs are arranged in connected rings around the larger particles. A fast Fourier transform of Figure 1c, shown in the inset in Figure 1d, confirms the nearly perfect hexagonal symmetry of the polymer particle array. The mean center-to-center distance of the polymer particles is $351 \pm 2 \mathrm{~nm}$, which is only slightly greater than the distance found in the absence of the AuNPs (see Table S1 and Figure S1 in the Supporting Information). Hence, we conclude that the polymer particles are in close contact, and the AuNPs are resting in the valley space between particles, with no more than a single AuNP layer separating the larger polymer particles. In the following discussion, we describe the AuNP arrangement as a nanogrid.

Rutherford backscattering (RBS) analysis was conducted to determine the vertical distribution of the AuNPs within the films (normal to the substrate). Figure le shows that there is a high concentration of $\mathrm{Au}, c_{\mathrm{Aw}}$ at the top surface in the nanogrid, above a depleted layer, which is attributed to the first layer of polymer particles. The $10 \mathrm{~nm}$ AuNPs are small enough to occupy the voids between the close-packed polymer particles in the underlying layers. The first three layers of polymer particles can be identified through the oscillation in $c_{\mathrm{Au}}$ (labeled as $\mathrm{A}-\mathrm{C}$ in Figure 1e). The spacing between the marked peaks is on the same order of magnitude as the polymer particle diameter. Deeper layers cannot be identified because of energy straggling effects of the ions. The labels (i-iv) mark the positions of AuNP enrichment, which are presumed to correspond to the $2 \mathrm{D}$ nanogrid 

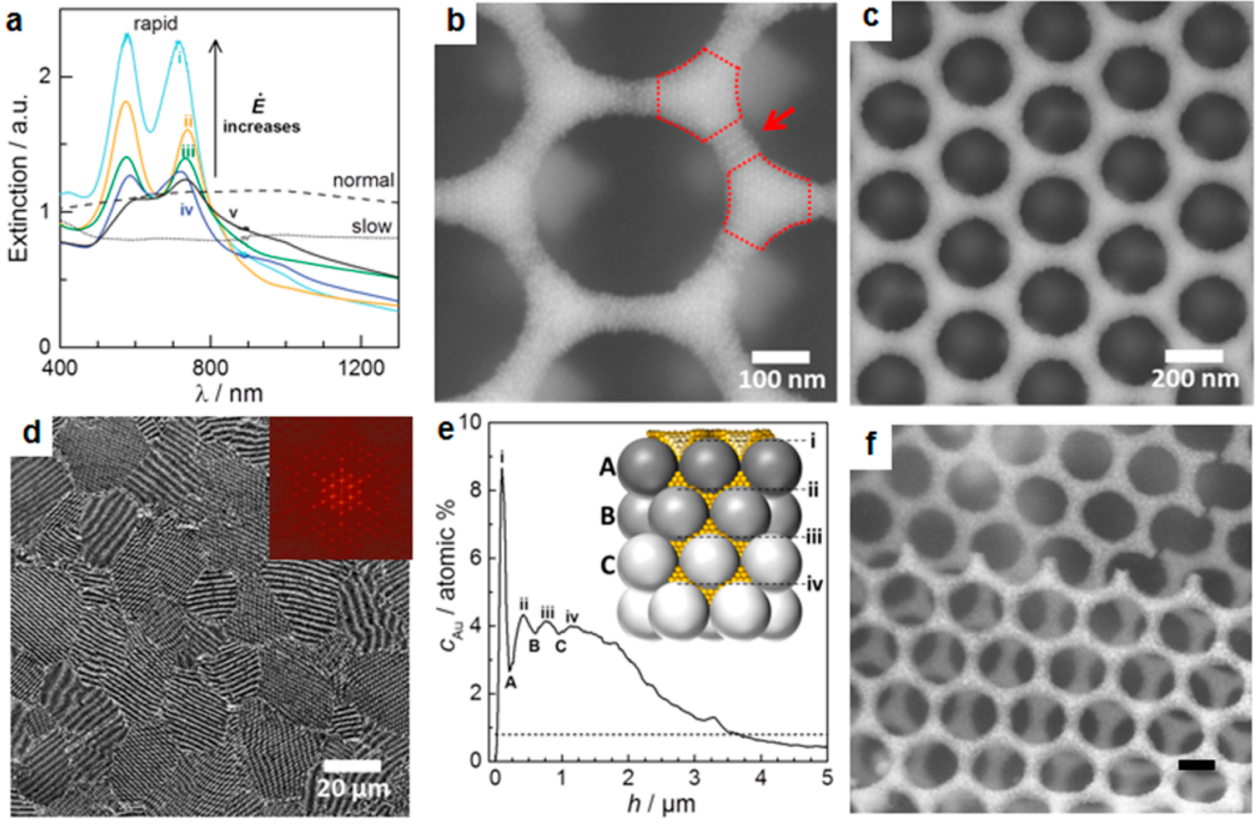

Figure 1. (a) UV-vis-NIR spectra of Au/polymer hybrid films fabricated with decreasing $\dot{E}$ : (i) 2.08; (ii) 1.81; (iii) 0.93 ; (iv) 0.67 ; and (v) 0.36 $\left(\right.$ all $\left.\times 10^{-6} \mathrm{~m} \mathrm{~s}^{-1}\right)$. The spectra for films normally dried in the ambient atmosphere $\left(---, \dot{E}=1.1 \times 10^{-7} \mathrm{~ms}^{-1}\right)$ and slowly dried under high humidity $\left(\cdots, \dot{E}=3.2 \times 10^{-9} \mathrm{~ms}^{-1}\right)$ are shown for comparison. (b) SEM image of a rapidly dried film $\left(\dot{E}=2.08 \times 10^{-6} \mathrm{~m} \mathrm{~s}^{-1}\right)$ at the highest magnification where individual AuNPs are visible. The AuNPs crystallize into truncated triangular-like shapes (outlined with a dashed line) and connected by bridges (indicated by the arrow). Scale bar is $100 \mathrm{~nm}$. (c) At lower magnifications, it is apparent that the larger polymer particles are in an ordered hexagonal array and the AuNPs create a grid-like structure. Scale bar is $200 \mathbf{~ n m}$. (d) At the lowest magnification, the formation of grain boundaries between the $2 \mathrm{D}$ crystals is visible. Scale bar is $20 \mu \mathrm{m}$. Inset: The fast Fourier transformation (FFT) of the nanogrid structure in (c) shows clearly that a hexagonal symmetry is well established. (e) Au concentration depth profile obtained from RBS analysis of a rapidly dried structure $\left(\dot{E}=2.08 \times 10^{-6} \mathrm{~m} \mathrm{~s}^{-1}\right)$. The high Au concentration at the surface $(>8$ at. \%) represents the nanogrid layer (labeled as i on the profile and on the inset diagram). The labels on the concentration profile mark the first three layers of polymer particles (A-C) in a closed-packed FCC crystal and the AuNP-enriched voids (ii-iv), as schematically illustrated in the inset diagram. The dashed line represents the concentration corresponding to a hypothetical homogeneous distribution of the AuNPs. Note: The inset diagram is not drawn to scale. (f) Examples of freestanding grid structures obtained from sintered AuNPs after dissolving the polymer phase. Scale bar is $200 \mathrm{~nm}$.

layer at the top and the planes of AuNPs filling the interparticle space between the hexagonally packed layers, as is schematically illustrated in the inset diagram. The wavelength of the oscillations in the Au concentration closely matches the spacing of the hexagonally packed layers in a colloidal crystal of $338 \mathrm{~nm}$ particles. We found that if the structure was heated at $340^{\circ} \mathrm{C}$ in a convection oven for $7 \mathrm{~min}$ to sinter the AuNPs, the polymer phase could thereafter be dissolved in toluene to leave a freestanding nanogrid sheet, as is shown in Figure 1f.

In summary, the results of the RBS analysis in combination with scanning electron microscopy (SEM) indicate a hybrid structure, which consists of a 3D binary colloidal crystal (closepacked polymer particles in which the interstitial space contains AuNPs) with a $2 \mathrm{D}$ nanogrid structure at the surface. The mechanism of the structure formation is considered next.

Influence of Evaporation Rate on Particle Ordering and Structuring. The vertical distribution of colloidal particles in a drying layer is determined by a competition between evaporation, which sweeps up particles at the top surface, and diffusion, which redistributes the particles evenly. For a film with an initial thickness of $H$, the rates of the two processes are compared via a Péclet number, given as $\mathrm{Pe}=H \dot{E} / D_{S}$, where $D_{\mathrm{S}}$ is the Stokes-Einstein diffusion coefficient of the particles. ${ }^{25}$ Only when $\mathrm{Pe}>1$, and evaporation is fast relative to diffusion, will particles accumulate near the top of the drying film surface. When $\mathrm{Pe}<1$, diffusion is fast relative to evaporation, and hence the particles will be distributed uniformly in the direction normal to the substrate during the drying process. $D$ is inversely related to particle size through the Stokes-Einstein equation, which means that slower-diffusing large particles are more prone to be found at the surface of a drying film compared to small particles.

In blends of large and small particles, each population of particles has a Péclet number, represented as $\mathrm{Pe}_{\mathrm{L}}$ and $\mathrm{Pe}_{\mathrm{S}}$, respectively. In the regime where $\mathrm{Pe}_{\mathrm{S}}<1<\mathrm{Pe}_{\mathrm{L}}$, a diffusive model ${ }^{26}$ predicts that stratification of particles by size will occur, with a greater concentration of large, slow-diffusing particles developing at the top surface. However, our experiments using NIR-assisted heating, in which $\dot{E}$ is fast, are in the regime where $\mathrm{Pe}_{\mathrm{L}}$ and $\mathrm{Pe}_{\mathrm{S}}$ both have values well above 1 . Values of $\mathrm{Pe}_{\mathrm{S}}$ range from 3.4 to 17.7 (using the hydrodynamic radius and the relevant drying temperatures), and $\mathrm{Pe}_{\mathrm{L}} / \mathrm{Pe}_{\mathrm{S}}=R_{\mathrm{L}} / R_{\mathrm{S}}$. Consequently, the polymer particles and AuNPs are both predicted to accumulate at the top surface during drying. Furthermore, according to the model developed by Cardinal et al., ${ }^{27}$ the sedimentation of particles on the time scale of drying is not significant in the experiments reported here.

SEM analysis of the surfaces of the films shows that periodic grid-like structures are formed over a range of evaporation rates obtained under NIR radiation for three sizes of polymer particles (mean diameters of 147, 261, and $338 \mathrm{~nm}$ ). (See Figure S2 in the Supporting Information.) As $\dot{E}$ is increased, the quality of the periodic ordering, determined from quantitative image analysis, likewise increases. (See Figure S3 in the Supporting Information.) The same positive correlation between the structure ordering and the evaporation rate was obtained for all three particle size ratios (32.5:1, 25.1:1, and 14.1:1). A simple geometric argument 
a

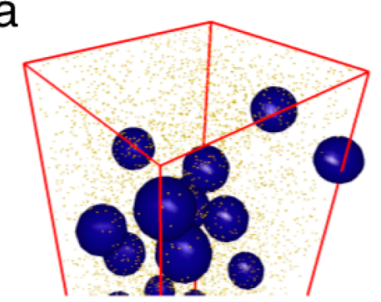

d

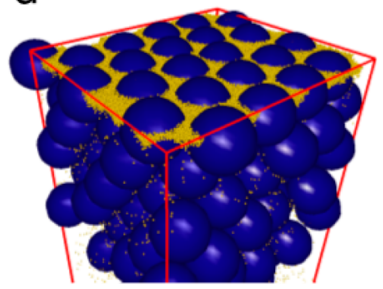

b

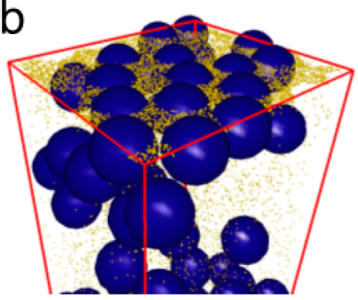

e

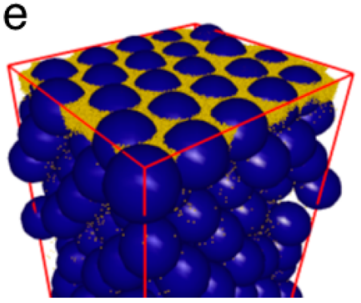

C

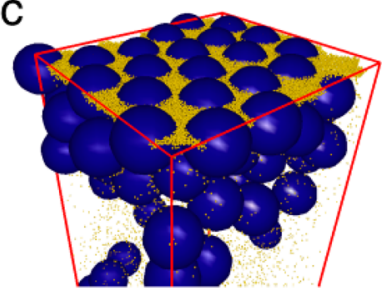

f

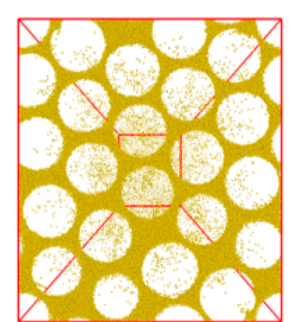

Figure 2. Simulation snapshots showing the particles accumulating at the model air-water interface with an evaporation rate $\dot{E}=12 R_{\mathrm{S}} / \tau_{\mathrm{B}}$. Here, $\tau_{\mathrm{B}}=4 R_{\mathrm{S}}^{2} / D_{\mathrm{S}}$ is the simulation time unit; it takes of order one unit of time for a small particle to diffuse a distance equal to its own radius. The radius of the large (blue) particles is 32 times greater than the small (yellow) particles. We calculate $D_{S}$ for the AuNPs in our experiments to be $1.1 \times 10^{-12} \mathrm{~m}^{2} \mathrm{~s}^{-1}$ such that $\tau_{\mathrm{B}} \approx 420 \mu \mathrm{s}$. (a) At time $t=0$, the system is at equilibrium and evaporation is started. (b) At time $t=50 \tau_{\mathrm{B}}$, both species are accumulating at the interface. (c) At time $t=130 \tau_{\mathrm{B}}$, the large particles start to form ordered layers at the interface. (d) At time $t=210 \tau_{\mathrm{B}}$, the large particles have formed crystalline layers at the interface, with small particles filling the interstitial spaces. (e) At time $t=410 \tau_{\mathrm{B}}$, the structure formed by the large particles has not changed, but the layer of small particles has increased its thickness. (f) Top view of the nanogrid of small particles at $t=410 \tau_{\mathrm{B}}$.

shows that small particles can pass through the holes in a plane of a close-packed array of larger particles, ${ }^{26}$ provided that the latter are larger by a factor of $(2 / \sqrt{3}-1)^{-1} \approx 6.5$. However, for particle blends with a size ratio of 14.1:1, the ring-like structures of the nanogrid are found only with evaporation rates above ca. $1 \times 10^{-6} \mathrm{~m} \mathrm{~s}^{-1}$ (Figure S2). At lower rates, the AuNPs appear only in subsurface interparticle spaces. For this blend, in which there is a greater geometric restriction, a faster evaporation rate is required for the AuNPs to be forced to the surface.

The surface structures of normally dried films, which lacked any strong optical extinction, were also examined. In this experiment, $\mathrm{Pe}_{\mathrm{S}}$ is $c a$. 1 , which is the tipping point below which the AuNPs will not accumulate at the top of the drying film. To achieve the lower evaporation rate, the dispersion was dried at room temperature, which is below the glass transition temperature, $T_{\mathrm{g}}$, of the polymer. Hence, the polymer particles are not deformed from their spherical shape, and they do not coalesce. A representative SEM image (Figure S4a, Supporting Information) shows that only a small number of AuNPs are seen on the film surface, appearing in the images as small bright spots. With slow drying, achieved under high humidity, $\mathrm{Pe}_{\mathrm{S}}$ takes a low value of 0.03. In this regime, AuNPs appear only in a few isolated domains and in chains along colloidal crystal boundaries (Figure S4b). Nanogrid structures do not develop. The Au concentration profile with a normal evaporation rate shows AuNP depletion in the first micrometer beneath the surface and then approaches the expected bulk concentration. (See the RBS analysis in Figure S4c of the Supporting Information.) With slow drying, there is a very low concentration of $\mathrm{Au}$ at the surface, and it gradually increases in concentration over a depth of $4 \mu \mathrm{m}$ into the surface. Only in the high-Pe regime obtained with NIR heating are the AuNPs found to accumulate in a nanogrid array at the top surface.

Simulations of the Structure Formation during Drying. We use Langevin dynamics simulations for particles in a solvent to explain the formation of the nanogrid of AuNPs. The Supporting Information provides details of the simulations. We model evaporation by a moving wall that pushes the particles toward the underlying substrate. Figure $2 a-e$ illustrate the evolution of the system for a blend of large and small particles with a size ratio $R_{\mathrm{S}}: R_{\mathrm{L}}=32: 1$ (similar to our experiments). Both species accumulate at the air/water interface, but the large particles form crystalline layers, while the small particles fill the interstitial spaces. The crystal of large particles grows downward from the surface, while the thickness of the layer of small particles also increases with time (see Figure S5 in the Supporting Information). The small particles move readily through the voids between the large particles to reach the top surface. This is clear in Movie 1 in the Supporting Information. One can think that the downward moving crystal acts as a "sieve" for the small particles, which move up through the holes in the sieve, to create the periodic nanogrid array shown in Figure $2 \mathrm{f}$, which appears very similar to the experimental structure in Figure 1c.

Our simulations neglect the forces due to the hydrodynamic flow of the evaporating water, but we obtain very similar nanostructures in experiment and simulation. The nanogrid appears to be a robust result, which is insensitive to flow forces. Our mechanism simply relies on particles of both sizes being pushed toward the surface. The downward movement of the air/water interface and the flow forces both push the particles in the same way.

Control of Surface Topography via the Evaporation Rate. With the aim of explaining the difference in the optical properties when the evaporation rates are changed, the surface structures were examined quantitatively by atomic force microscopy (AFM). In the AFM images presented in Figure $3 a-c$, the surface morphology of the films is seen to change from a convex to a concave structure as the evaporation rate is increased. The transition in the surface morphology is attributed to the increasing velocity of the downward moving sieve, consisting of the close-packed polymer particles. The evaporation rates set the velocity at which the sieve drops downward. At a low velocity of the sieve, the AuNPs are pushed through the holes in the sieve and start to fill the interparticle spaces between the dome-like polymer particles (Figure 3a). When the velocity of the 

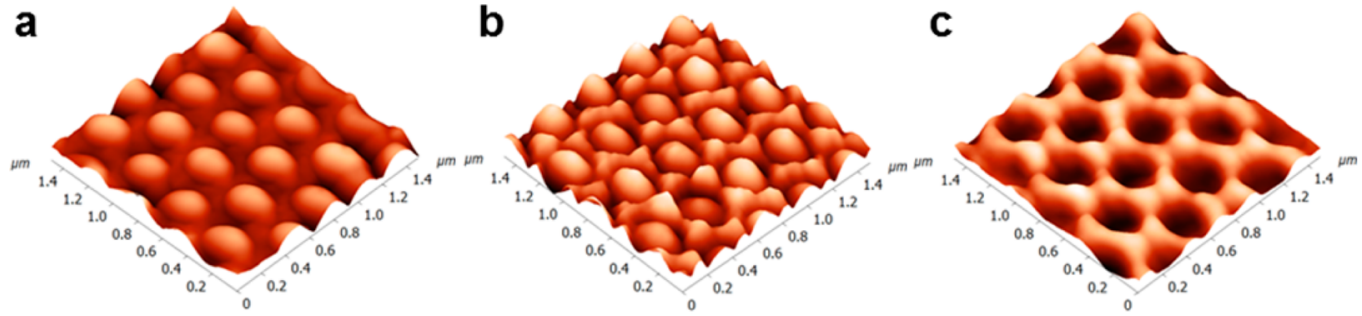

$$
\dot{E}=3.5 \times 10^{-7} \mathrm{~m} \mathrm{~s}^{-1} \quad \dot{E}=9.3 \times 10^{-7} \mathrm{~m} \mathrm{~s}^{-1} \quad \dot{E}=20.8 \times 10^{-7} \mathrm{~m} \mathrm{~s}^{-1}
$$
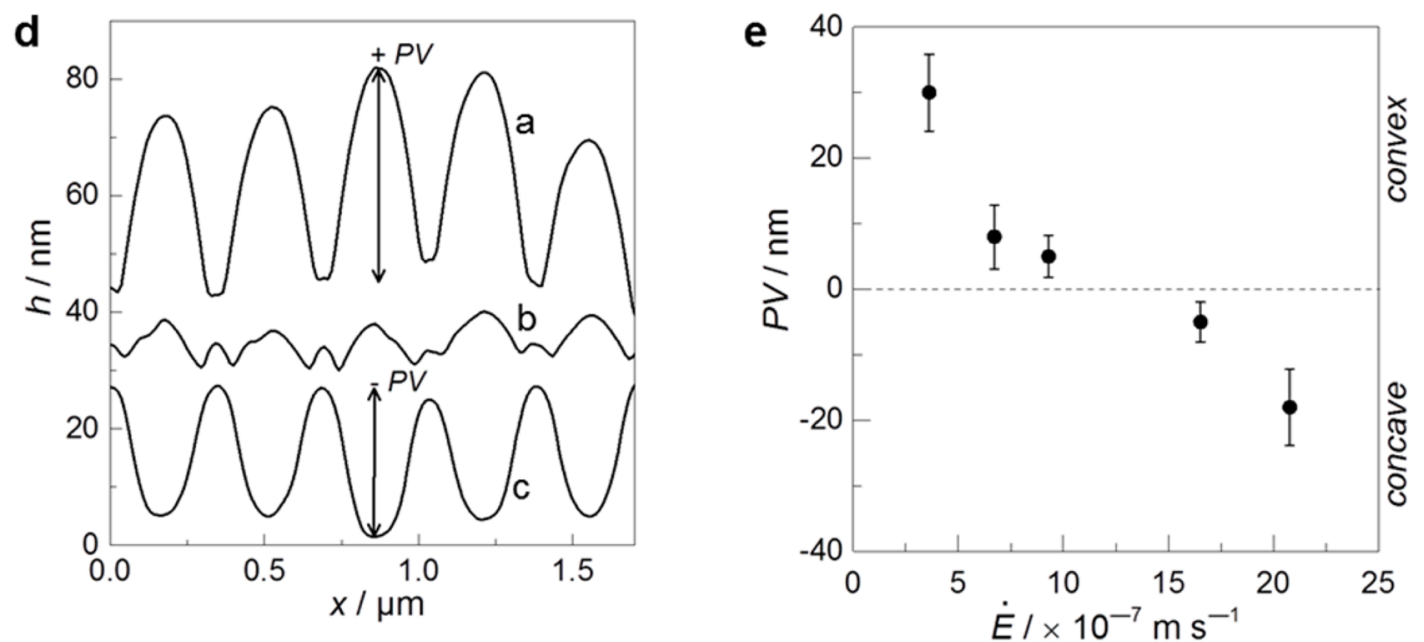

Figure 3. AFM topographic images to show the surface morphology as a function of the evaporation rate: (a) $3.5 \times 10^{-7} \mathrm{~m} \mathrm{~s}^{-1}$; (b) $9.3 \times$ $10^{-7} \mathrm{~m} \mathrm{~s}^{-1}$; and (c) $20.8 \times 10^{-7} \mathrm{~m} \mathrm{~s}^{-1}$. The scan size is $1.5 \mu \mathrm{m} \times 1.5 \mu \mathrm{m}$ for all three images. When $P_{\mathrm{s}}$ is increased from 3.4 to 17.7 (through an increase in $\dot{E}$ ), the $10 \mathrm{~nm}$ AuNPs pile up and build a protruding nanogrid structure. (d) Line traces of the surface topography for the three structures shown in a-c. (e) Plot of the peak-to-valley height of the film surface as a function of the evaporation rate.

downward moving sieve is increased, the AuNPs start to pile up on the film surface and exceed the level of the domes of the polymer particles (Figure 3b). Finally, at the highest velocity, the AuNPs crystallize into the truncated triangular-like shapes that are connected by bridges (Figure 3c), as were already shown in Figure $1 \mathrm{~b}$, resulting in a protruding grid nanostructure.

The corresponding 2D line traces are shown in Figure 3d. Here, the peak-to-valley height, PV, is defined as the difference in the height at the polymer particle center and at the polymer particle boundary. A positive PV is obtained for the convex structure formed by the domes of the polymer particles, and a negative PV arises from the concave honeycomb structure with the AuNPs protruding from the surface. As a means to quantify the changes in the surface morphology, PV is plotted as a function of evaporation rate (see Figure $3 \mathrm{e}$ ). As can be seen in this figure, PV decreases continuously with increasing $\dot{E}$. Faster evaporation rates lead to the AuNPs being pushed higher above the polymer particle surface. At slower evaporation rates, the sieve effect is weaker, and perhaps there is less upward flow. Hence, AuNPs are not pushed through the interstices of the larger polymer particles. These observations of the changing surface morphology present interesting routes to tailor the surface properties of a highly ordered nanostructure simply by changes in the evaporation rate and without the need for any sophisticated equipment or multiple processing steps.

An important finding is that with the fastest evaporation rates achieved with NIR heating, the arrays of AuNPs protrude upward from the surface. In turn, the protruding nanogrid structure is a requirement for the observed optical signatures, which are described in detail next.
Optical Response of Nanogrids. The optical properties of the optimized nanogrid structures were investigated using broadband angle-dependent spectroscopy and optical microscopy. The optimized samples correspond to the structures that were dried rapidly, with the highest evaporation rate of $\dot{E}=2.08 \times$ $10^{-6} \mathrm{~m} \mathrm{~s}^{-1}$.

A microscope spectrometer setup was used to map the reflection across an area of $20 \mu \mathrm{m} \times 20 \mu \mathrm{m}$. In these scans, an area including both the nanogrid structure and grain boundaries was selected when viewing with a CCD camera. The dark-field image, obtained with this camera, is presented in Figure 4a. The grain boundaries can be easily identified through the strong reflection of the incident white light; the scanned area is indicated by the red box. The scans were performed at three fixed wavelengths of 570,750 , and $890 \mathrm{~nm}$. These wavelengths of interest were selected because they are close to the maximum peak positions in the extinction and reflection spectra in Figure 1a. The reflection map for $\lambda=570 \mathrm{~nm}$ shows generally a weak reflection except for some regions in the grain boundaries (Figure $4 b$ ). At $\lambda=750 \mathrm{~nm}$ the overall reflection starts to increase, as can be seen in Figure 4c. At $\lambda=890 \mathrm{~nm}$, only the hybrid structure shows a strong reflection (Figure 4d).

Spectroscopic information was collected at two spots in the 2D image corresponding to the center of the hybrid colloidal crystal/ nanogrid domain and the grain boundary. Good agreement is obtained between the microscopic spectra of the hybrid and the macroscopic UV-vis spectra (Figure 1a) in the positions of the peaks. Differences are visible in the values for extinction intensities. These differences can have their origin in the difference in collection angles for the UV-vis and the microscopy setup, the 

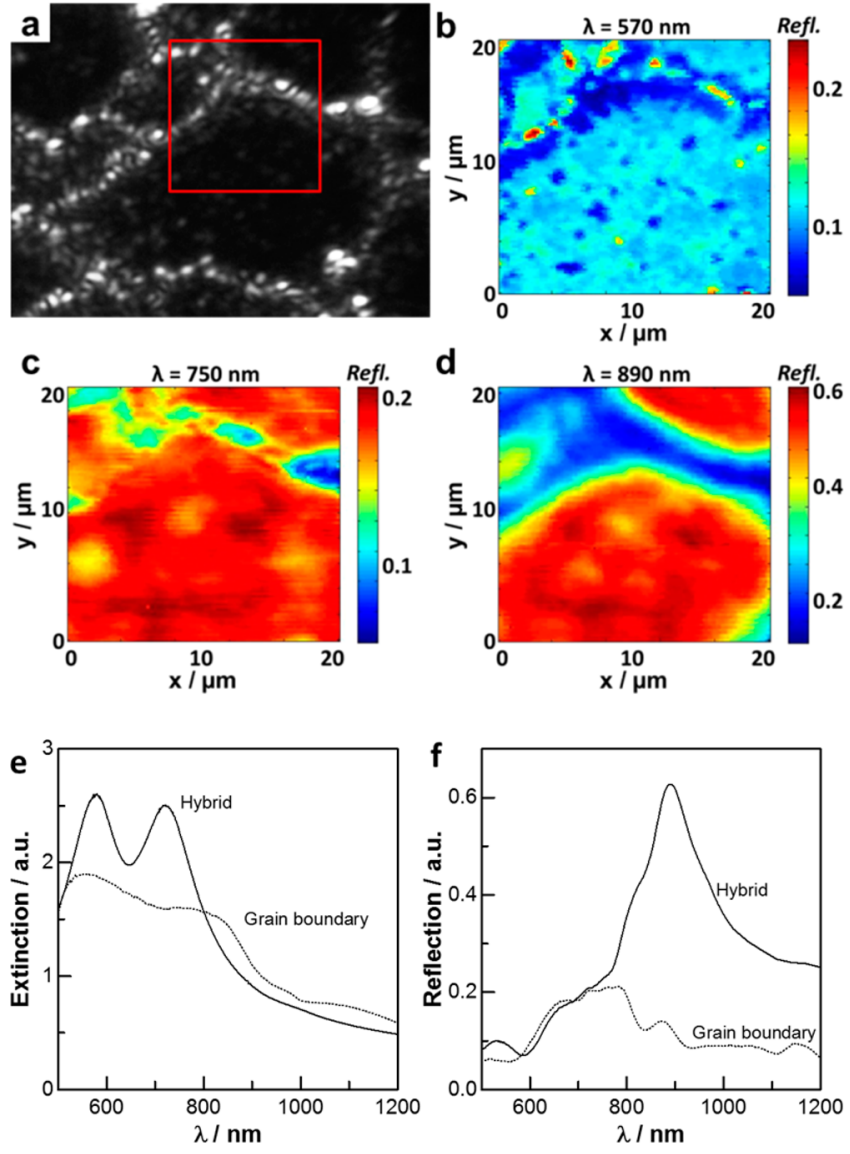

Figure 4. Optical reflection microscopy maps. (a) Dark-field micrograph of the film surface with grain boundaries, which appear bright. The $20 \mu \mathrm{m} \times 20 \mu \mathrm{m}$ scanned area is indicated by the red selection. Optical reflection microscopy maps at wavelengths of (b) $570 \mathrm{~nm}$, (c) $750 \mathrm{~nm}$, and (d) $890 \mathrm{~nm}$. The color indicates the reflectivity: red is strongly reflecting, and blue is poorly reflecting. (e) Optical extinction (transmission) and (f) reflectivity spectra corresponding to $<1 \mu \mathrm{m}^{2}$ areas in the hybrid nanogrid crystal and grain boundaries, as labeled.

latter collecting a larger fraction of near-forward scattered light. Figure $4 \mathrm{e}$ and $\mathrm{f}$ show that the extinction spectrum with the two peaks is characteristic for the hybrid crystal structure. Also the reflection spectrum shows a sharp peak (with a center wavelength of $\lambda_{\max }=890 \mathrm{~nm}$ ) only for the hybrid structure.

The position of the first-order stopband for the opal photonic crystal is given by the condition $a / \lambda \approx 0.6$, where $a=\sqrt{2} d$ is the lattice period, with $d=2 R_{\mathrm{L}}$, the colloidal polymer particle diameter. With $d=338 \mathrm{~nm}$, the stopband is expected at $797 \mathrm{~nm}$. The position for the photonic reflection from the hybrid structure is red-shifted (by ca. $100 \mathrm{~nm}$ ) compared to the expected value for the bulk because of the higher index of the nanovoids filled with AuNPs, which is in agreement with previous studies on AuNP-infiltrated opal photonic crystals. ${ }^{28,29}$ Also, because the AuNP infiltration is not uniform in depth, the gradient in the effective index is expected to be responsible for some of the broadening observed in the experimental peak. The position of the absorption band around $590 \mathrm{~nm}$ agrees well with the expected absorption band of closely packed AuNPs that is redshifted compared to the response of individual particles due to the strong dipolar interactions and aggregation effects. ${ }^{29}$ The origin of the second absorption feature around $750 \mathrm{~nm}$ is related to the array periodicity, as can be observed when changing the size of the nanogrid via the polymer particle size (see Supporting Information Figure S7). The peak can be attributed to the appearance of an additional stopband of the opal, mediated by the high permittivity of the AuNP effective medium. ${ }^{30}$

Plasmonic Resonances of the AuNP Nanogrid. The optical response of the AuNP nanogrid is very complex. Contributions are expected from a variety of light-matter interaction mechanisms, which strongly depend on the connectivity and geometry of the metallic constituents, ranging from (semi-) continuous plasmonic networks to isolated nanoparticles. ${ }^{28-37}$ When dilute suspensions of AuNPs begin to aggregate, the nature of the collective electronic resonances giving rise to the plasmonic response changes dramatically, and for a fully aggregated system, a red-shifted extinction peak is expected to dominate the spectral response. ${ }^{31}$ The detailed electromagnetic properties of the tightly packed AuNP aggregate are strongly dependent on their size and packing ratio as well as on the dielectric background surrounding the particles. ${ }^{32,33}$ In addition to the single-NP plasmonic resonances and the coupled plasmon modes induced by the hybridization of the NP resonances, we expect contributions from the localized plasmons formed in the metallic voids surrounding the polymer template and delocalized Bragg-like plasmons propagating in the three-dimensional $\mathrm{Au}$ network. ${ }^{34-38}$ The plasmonic response is then modulated by the photonic stopbands of the underlying photonic-crystal polymer template. $^{30,35,39}$

Because of the multiple length scales involved and the complexity of the dielectric function, numerical simulations of the exact system configuration are not feasible. We have modeled the AuNP nanogrid as a homogeneous medium with an effective dielectric constant defined by a renormalized Maxwell Garnett approach. ${ }^{40}$ This method allows the description of ensembles of resonant plasmonic particles even at high filling ratios and is in good agreement with direct finite difference time domain (FDTD) simulations. ${ }^{40}$ The modeling of the effective permittivity of the $\mathrm{Au}$ is described in the Supporting Information (Figures S8 and S9).

We have performed FDTD simulations, shown in Figure 5, to explore the plasmonic response of the structure. Figure 5a shows the results for the simulated reflection, transmission, and absorption through the structured nanogrid material. The reflection spectrum presents a number of peaks associated with the microstructured-Au layer reflectivity (around $630 \mathrm{~nm}$, which matches the maximum of the effective index of refraction of the $\mathrm{Au}$ for large filling ratios), the emerging full band gap of the $\mathrm{Au}$ inverted opal structure (around $780 \mathrm{~nm}$ ), and the expected $\Gamma-\mathrm{L}$ stopband $(870 \mathrm{~nm})$. The reflectivity also shows the characteristic finite-system oscillations (as the simulated opal structure consists of 12 layers). While the overall shape and peak position are in good agreement with the measurements in Figure $4 \mathrm{e}$ and $\mathrm{f}$, we note that the predicted reflection peaks at 630 and $780 \mathrm{~nm}$ appear only as weakly defined shoulders in the overall measured reflectivity.

This smearing of the measured reflection peaks is attributed mainly to the imperfections in the fabricated structure and the relatively small size of the system that was simulated. As is expected, the emerging full photonic band gap associated with the peak at $780 \mathrm{~nm}$ is affected the most. This is because the full band gap is a higher order gap, which opens between the eighth and ninth bands of the inverted opal structure and is strongly affected by small-scale fluctuations in the dielectric structure. Our observations are in line with those found for the nanoparticlebased metallodielectric inverse opals described by Wang et al. ${ }^{39}$ 

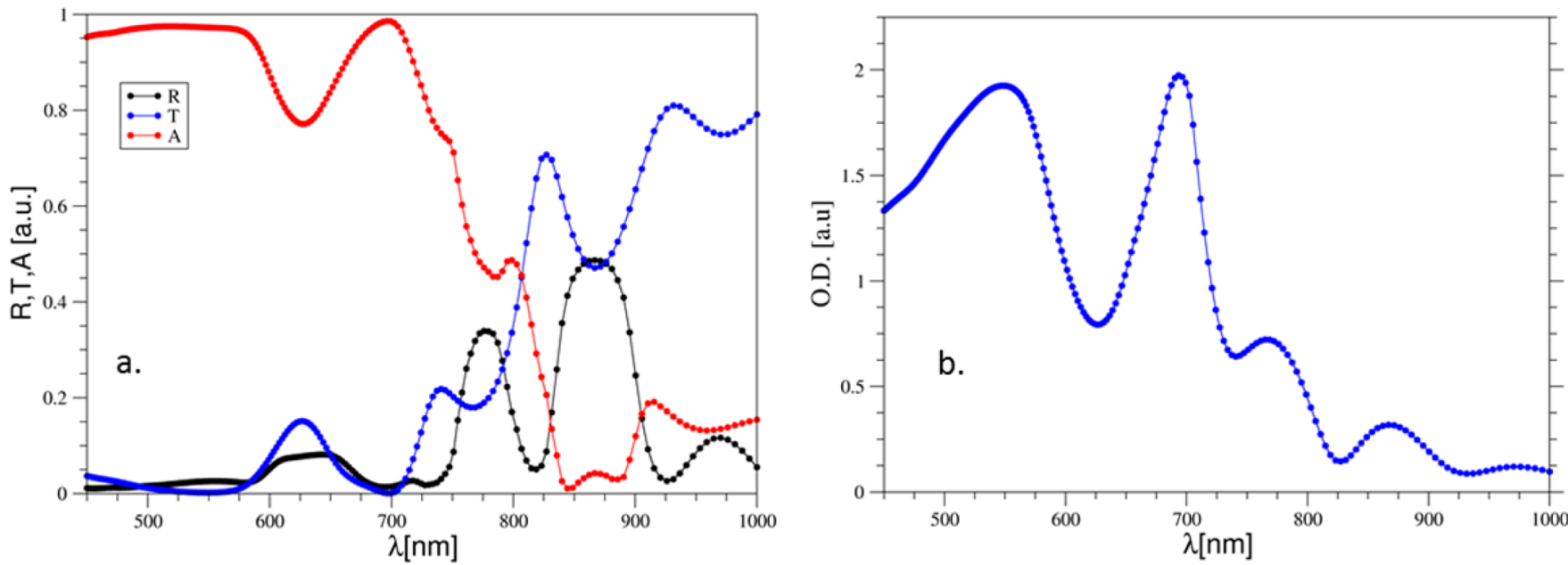

Figure 5. (a) FDTD simulations of the reflection (R), transmission (T), and absorption (A) for a 12-layer Au inverted opal structure with a protruding nanogrid Au layer. (b) Extinction spectrum calculated from the optical density defined as $O D=-\log (T)$.
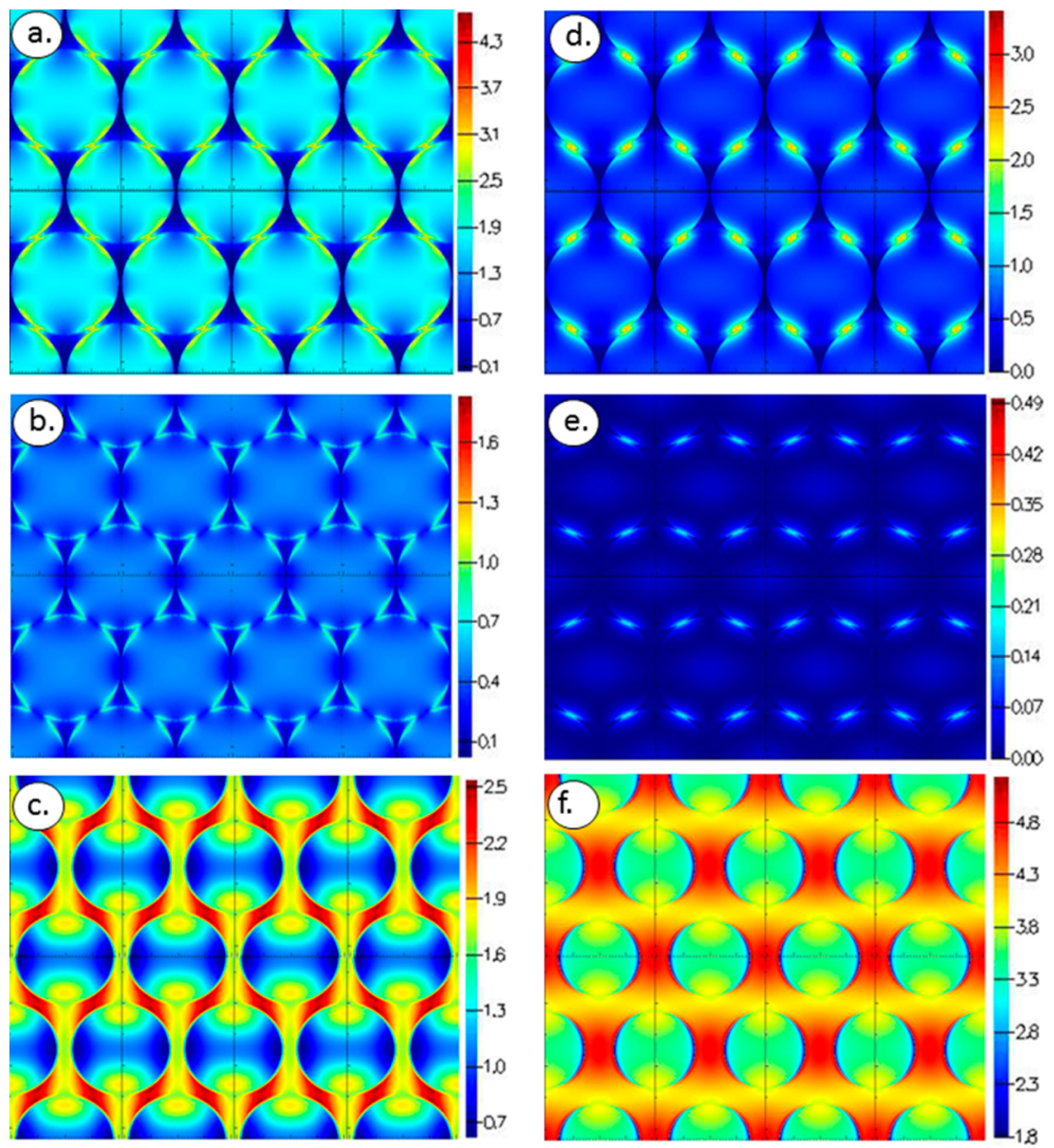

Figure 6. Simulations of the magnitude squared of the electric field. $(\mathrm{a}-\mathrm{c})$ Plots of the field associated with the left peak at $550 \mathrm{~nm}$ in Figure $5 \mathrm{~b}$ and $(\mathrm{d}-\mathrm{f})$ the fields associated with the right peak at $696 \mathrm{~nm}$, respectively. The fields are plotted at three depths from the surface within the 3D hybrid structure: (a and d) at a depth of $30 \mathrm{~nm}$; (b and e) at $120 \mathrm{~nm}$; and (c and f) at a depth of $1050 \mathrm{~nm}$.

The absorption spectrum presents a broad peak in the lower part of the spectrum induced by the hybridized NP resonances and bulk $\mathrm{Au}$ absorption and a second major peak at around $700 \mathrm{~nm}$. The origin of the second peak is related to a structuringinduced plasmonic relaxation, as is confirmed by the electric field distributions shown in Figure 6. In this case, the flatness of the photonics bands associated with the emergent photonic band gap causes a strongly reduced group velocity. ${ }^{37}$ The subsequent slow-light effects result in a strong enhancement of the plasmonic absorption, similar to effects found in previous works. ${ }^{37,40}$ The extinction spectrum in Figure $5 \mathrm{~b}$ displays two major peaks, at 550 and $696 \mathrm{~nm}$. Although it has an overall shape and peak 
position similar to the measurement in Figure 4e, the peaks are blue-shifted due to the approximations made when evaluating the effective Au dielectric permittivity.

We have also analyzed the electric field distribution in the $\mathrm{Au}$ nanogrid structure. Figure 6 shows the squared magnitude of the electric field at 550 and $696 \mathrm{~nm}$ for three different depths inside the structure: $30 \mathrm{~nm}$ in the protruding nanogrid above the opal structure, $120 \mathrm{~nm}$ inside the first layer of the inverted opal, and $1050 \mathrm{~nm}$ at the bottom of the third layer of the inverted opal. The field distribution for the peak at $550 \mathrm{~nm}$ suggests a delocalized mode with the electric field modulated along the inverted opal structure, which is consistent with the excitations of the hybridized AuNP resonances throughout the Au fraction. In contrast, the distribution of the field for $696 \mathrm{~nm}$ suggests a strongly localized mode with well-defined hot spots. As the depth of the observation is increased, the Au filling is reduced and eventually the field becomes more uniformly distributed. Furthermore, the tightly localized field for the $696 \mathrm{~nm}$ mode is strongly affected by the structure filling fraction. In the absence of a well-defined, tightly packed protruding AuNP layer, it vanishes, which is consistent with the experimental findings (Figures $1 \mathrm{a}$ and $4 \mathrm{e}$ ). The protruding AuNP nanogrid structure is found only in experiments using the faster evaporation rates, and it is only these structures that exhibit two extinction peaks. To conclude, the overall optical response of the structure is determined by both the nanoengineering of the Au effective dielectric permittivity and the microstructuring of the inverted opal structure. Our simulations support the key experimental finding of two extinction peaks.

\section{CONCLUSIONS}

We have developed a simple and fast method of colloidal self-assembly to fabricate a hybrid structure of a quasi-2D array of $\mathrm{Au}$ nanoparticles on a $3 \mathrm{D}$ opal structure. We use a horizontal deposition process assisted by NIR radiation to achieve the required fast evaporation rates. The strongest optical extinction, which is associated with the greatest level of order and a protruding Au nanogrid, arises from the fastest evaporation rates. Langevin dynamics simulations reveal that, with fast evaporation rates when the particles are swept up by the falling meniscus, the Au nanoparticles pass through the interstitial spaces of the larger polymer particles. The use of NIR radiative heating not only leads to very short fabrication times (about $7 \mathrm{~min}$ ) but also yields unusual optical spectra. With simulations, we trace the double peaks in the measured extinction spectra to elementary plasmonic resonances compounded by the optical response of the supporting opal structure. The optical properties can be tuned through the power density of the NIR radiation used to speed the evaporation rate. Our method overcomes the time limitations of a conventional horizontal deposition method while being low-cost and possible to apply over areas greater than several $\mathrm{cm}^{2}$.

\section{METHODS}

Synthesis of the Particles. In the synthesis of AuNPs all chemicals were purchased from Sigma-Aldrich and used without additional purification. The well-established Turkevich method ${ }^{41}$ was used. A $100 \mathrm{~mL}$ amount of a $1 \mathrm{mM} \mathrm{NaAuCl} \mathrm{C}_{4}$ aqueous solution was brought to the boil, followed by the addition of $5 \mathrm{~mL}$ of a preheated trisodium citrate aqueous solution ( $2 \mathrm{wt} \%$ ) under vigorous stirring. After $5 \mathrm{~min}$ the color of the reaction solution turned to a characteristic red. The stirring was slowed, and the mixture was boiled for a further $15 \mathrm{~min}$ before cooling to room temperature.
To enable the purification of the AuNPs by centrifugation and to increase the stability of the dispersion against aggregation, the nanoparticles were coated with bis( $p$-sulfonatophenyl)phenyl-phosphine dihydrate dipotassium salt (BSPP) by a ligand exchange reaction. ${ }^{42}$ To $100 \mathrm{~mL}$ of a dispersion of the original citrate-coated AuNPs was added $50 \mathrm{mg}$ of BSPP. After stirring for at least $4 \mathrm{~h}$, the reaction mixture was passed through a $0.45 \mu \mathrm{m}$ filter (Millipore). A saturated sodium chloride solution was added to the AuNP dispersion until the color changed from red to blue, indicating the formation of aggregates. The dispersion of the aggregates was centrifuged for $10 \mathrm{~min}$ at $5000 \mathrm{rpm}$. The supernatant, containing the excess BSPP, citrate, and sodium chloride, was discarded. The black precipitate was redispersed in $1 \mathrm{~mL}$ of purified water and sonicated. The concentration of the final AuNP dispersions was determined using the method by Haiss et al. ${ }^{43}$

A dispersion of the acrylic copolymer particles was synthesized at AkzoNobel Decorative R\&D (Slough UK). The particles, composed of a copolymer of methyl methacrylate, butyl acrylate, and methacrylic acid (in a ratio of 18.3:13.3:1 by weight), were made by semicontinuous emulsion polymerization using an anionic, ethoxylated alcohol surfactant (Rhodafac RK500A, Rhodia) with ammonium persulfate as the initiator. In previous work, we reported the evaporation-driven transport in blends of AuNPs and these polymer particles, in the direction parallel to the substrate. ${ }^{22}$

Characterization of the Particles. The true diameter, $d$, of the AuNPs was determined by transmission electron microscopy (TEM) with a FEI Technai 12 microscope operated at $80 \mathrm{kV}$. The true diameter of the acrylic copolymer particles was determined by SEM with a JEOL $7100 \mathrm{~F}$ field-emission scanning electron microscope operated at an accelerating voltage of $3 \mathrm{kV}$. The hydrodynamic diameter, $d_{\mathrm{hyd}}$, was measured by dynamic light scattering using a Malvern ME11 Zetasizer Nano ZS. The same instrument was used to measure the zeta-potential ( $\zeta$-potential) of the particles. The glass transition temperature, $T_{g}$, of the dry polymer particles was $37.9^{\circ} \mathrm{C}$ measured by differential scanning calorimetry (Q1000, TA Instruments). The results of the particle characterization are summarized in Table 1 . The values for the true

Table 1. Sizes and $\zeta$-Potential of the Acrylic Polymer Particles and AuNPs

\begin{tabular}{lccc}
\multicolumn{1}{c}{ composition } & $d(\mathrm{~nm})$ & $d_{\mathrm{h}}(\mathrm{nm})$ & $\zeta$-potential $(\mathrm{mV})$ \\
acrylic copolymer & $338 \pm 5$ & $400 \pm 6$ & $-69 \pm 1$ \\
acrylic copolymer & $261 \pm 5$ & $307 \pm 6$ & $-68 \pm 1$ \\
acrylic copolymer & $147 \pm 6$ & $174 \pm 1$ & $-67 \pm 1$ \\
$10 \mathrm{~nm}$ AuNPs & $10.4 \pm 1$ & $21.1 \pm 1$ & $-50.4 \pm 2$ \\
\hline
\end{tabular}

diameter, $d$, are always lower than the hydrodynamic diameter, $d_{\mathrm{h}}$ because the latter is related to the effective size of the diffusing object.

Film Preparation and Drying. The films were prepared by casting aqueous polymer/AuNP blends with a micropipet on clean microscope glass coverslips $(18 \mathrm{~mm} \times 18 \mathrm{~mm})$ or appropriately sized square pieces of a silicon wafer. In all experiments, the initial film thickness of the wet film, $H$, was $900 \mu \mathrm{m}$ and the initial volume fraction of the solid polymer was $4.4 \%$, so as to obtain a film with a final thickness of $40 \mu \mathrm{m}$. The volume fraction of the AuNPs in the dry films was fixed as $0.32 \mathrm{vol} \%$ in all experiments. The films were dried under one of three different types of condition: (1) "rapid drying" through radiation with a near-IRemitting lamp, (2) "normal drying" at room temperature and in static air $\left(T=21^{\circ} \mathrm{C}, \mathrm{RH}=50 \%\right)$, leading to $\dot{E}=1.1 \times 10^{-7} \mathrm{~ms}^{-1}$, and (3) "slow drying" in a high-humidity atmosphere $\left(T=23^{\circ} \mathrm{C}, \mathrm{RH}\right.$ typically $\left.>95 \%\right)$, leading to $\dot{E}=3.2 \times 10^{-9} \mathrm{~ms}^{-1}$.

A chamber, made of acrylic sheets, was used to create the highhumidity environment. A beaker filled with water on a hot-plate (at $40^{\circ} \mathrm{C}$ ) was placed inside the box in order to create a high-humidity atmosphere. A small rotary fan was placed beneath the ceiling of the box to prevent condensation of water. With this setup, an atmosphere with a relative humidity of $>95 \%$ was created inside the chamber, resulting in a film drying time of 6 days. To measure the evaporation rate of water, a digital balance (interfaced with a computer to record data) was used inside the chamber. 
NIR Radiation-Assisted Drying and Evaporation Rate Determination. For fast drying, the wet films were placed beneath a $250 \mathrm{~W}$ near-IR-radiation-emitting lamp (model $470 \mathrm{IR}$, Interhatch). The experimental setup is shown in Figure S10. The wet films were placed with a certain distance, $d_{\mathrm{L}}$, from the near-IR lamp on a heat-shielded balance and with a thermocouple inside to record changes in mass and temperature over time. The gravimetric measurement recorded an evaporation rate, $E$, in units of mass per unit of time per area of the film (found from the slope in Figure 7a). An evaporation rate expressed as a
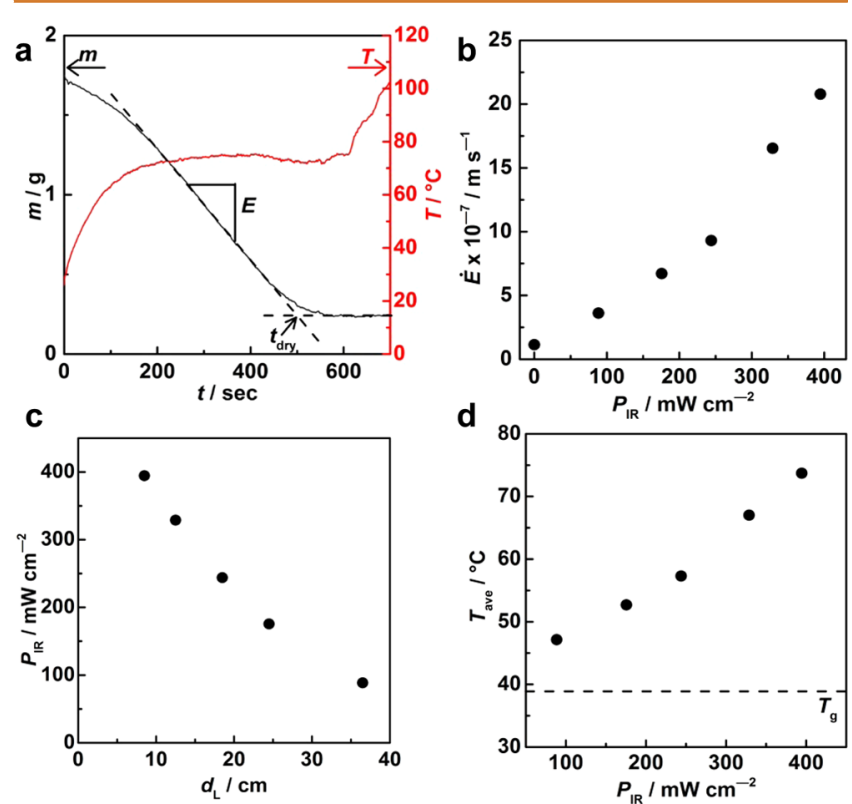

Figure 7. Infrared-radiation-assisted rapid drying and determination of the evaporation rate. (a) Changes in mass and temperature over time for a typical experiment. (b) Evaporation rate increases with $P_{\mathrm{IR}}$. (c) In turn, $P_{\mathrm{IR}}$ can be adjusted through variation of $d_{\mathrm{L}}$. (d) Average temperature, $T_{\mathrm{av}}$, increases as a function of $P_{\mathrm{IR}}$. The dashed line indicates the $T_{\mathrm{g}}$ of the polymer particles.

velocity, $\dot{E}$, is obtained by dividing $E$ by the density of the liquid water, $\rho\left(1000 \mathrm{~kg} \mathrm{~m}^{-3}\right)$, and the surface area, $A$, to give $\dot{E}=E /(\rho A)$. Figure $7 \mathrm{~b}$ shows the evaporation rate increasing as the power density of the infrared radiation, $P_{\mathrm{IR}}$ increases. This parameter was increased by decreasing $d_{\mathrm{L}}$, as is shown in Figure $7 \mathrm{c}$. The Péclet number depends on the Stokes-Einstein (SE) diffusion coefficient, $D_{S}$, which, in turn, is a function of the temperature, $T$, via the SE equation. For the estimation of $D_{\mathrm{S}}$, an average temperature, $T_{\mathrm{av}}$, which is defined as the mean of all temperatures obtained at times between $t=0$ and the drying time, was used. $T_{\mathrm{av}}$ increases with $P_{\mathrm{IR}}$ as shown in Figure $7 \mathrm{~d}$. The dashed line shows the glass transition temperature, $T_{\mathrm{g}}$, of the polymer particles. $T_{\mathrm{av}}$ is always higher than $T_{\mathrm{g}}$, which enables the formation of a hard, cohesive film via particle coalescence. ${ }^{44}$

Characterization of Hybrid Nanogrids. Scanning electron microscopy was conducted on a field-emission electron microscope (JEOL 7100F FE-SEM). All samples were coated by a thin layer of carbon (>20 nm, in an Edwards Auto 306 coater) to increase their electrical conductivity. In the standard procedure, the SEM was operated with an accelerating voltage of $10 \mathrm{kV}$. When the AuNP concentration on the film surface was low, the acceleration voltage was reduced to $5 \mathrm{kV}$ in order to prevent charging effects and damage by the electron beam.

Atomic force microscopy images were obtained on an NTEGRA microscope (NT-MDT) in intermittent contact mode in air, using a silicon cantilever with a spring constant in the range from 10 to $130 \mathrm{~N} / \mathrm{m}$ and a nominal resonant frequency of $275 \mathrm{kHz}$. UV-vis-NIR spectroscopy was performed using a commercial spectrometer (Cray 5000, manufactured by Varian).
Rutherford backscattering spectrometry was conducted at the Surrey Ion Beam Centre using a $2 \mathrm{MV}$ Tandetron accelerator (High Voltage Engineering Europe). A $5.5 \mathrm{MeV}^{7} \mathrm{Li}^{2+}$ beam with good depth resolution was used for detailed structure analysis of the surface up to $4 \mu \mathrm{m}$ deep. The scan size was more than $1 \mathrm{~mm}^{2}$ with a spot size of $c a .10 \mu \mathrm{m}$. The particle detector and X-ray detector were placed at an angle of $163^{\circ}$ and $135^{\circ}$, respectively. ${ }^{45}$ To reduce the RBS data, the DataFurnace code ${ }^{46}$ was used with the software NDFv9.5f to fit the data. ${ }^{47}$

Microscopic UV-vis-NIR spectroscopy (transmission and reflection) was performed using the output power of a $2 \mathrm{~W}$ broadband supercontinuum laser (Fianium) as a light source. The light beam was coupled into a subtractive-mode double prism monochromator to select a narrow wavelength range of a few nanometers. To focus the output of the wavelength selector on the sample surface, the light beam was coupled into a near-infrared objective lens (Mitotoyu Plan Apo NIR, $100 \times$, NA 0.5). The transmitted or reflected beam was collected by photodetectors for the visible $(\mathrm{Si})$ and near-infrared (InGaAs) ranges. A closed-loop piezo-stage was used to move the sample through the focused beam in order to obtain a $20 \mu \mathrm{m} \times 20 \mu \mathrm{m} 2 \mathrm{D}$ transmission or reflection map of the sample.

Macroscopic UV-vis-NIR spectroscopy was performed using a commercial spectrometer (Cary 5000, manufactured by Varian) with a beam having a $1 \mathrm{~mm}$ diameter.

Langevin Dynamics Simulations. Langevin dynamics simulations used the LAMMPS simulation code ${ }^{48}$ customized to consider the downward moving water/air interface by modeling it as a soft wall. The parameters in the simulations were chosen to agree with the experiment; there were $2 \times 10^{5}$ particles with a number ratio $N_{\mathrm{S}}: N_{\mathrm{L}}=212: 1$ and a size ratio $R_{\mathrm{L}}: R_{\mathrm{S}}=32: 1$. The contact angle of the particles at the water surface was taken to be $\theta=75^{\circ}$. Other technical information is provided in the Supporting Information.

Simulations of Optical Properties of Hybrid Nanogrids. A finite-difference time-domain simulation was implemented using Lumerical Solutions software (FDTD solutions) to perform transmission and reflection calculations. The polymer opal was modeled as a 12-layer FCC lattice of spheres with a refractive index of 1.47, particle radius of $169 \mathrm{~nm}$, and FCC lattice constant of $478 \mathrm{~nm}$. The structure depth was set to approximately $3600 \mathrm{~nm}$ to balance accuracy with computational cost. We used a nonuniform mesh with a Lumericaldefined mesh quality factor of 8 (maximum), and the $\mathrm{Au}$ populated regions were meshed separately with a resolution of $1 \mathrm{~nm}$. The protruding nanogrid structure was constructed by etching down with a triangular layer of closed-packed spheres aligned to the top layer of polymer spheres through a $40 \mathrm{~nm}$ thick Au layer placed on top of the polymer opal. The structure is shown in Figure S9.

\section{ASSOCIATED CONTENT}

\section{Supporting Information}

The Supporting Information is available free of charge on the ACS Publications website at DOI: 10.1021/acsnano.5b06886. The authors confirm that data underlying the findings are available without restriction. Details of the data and how to request access are available from the University of Surrey publications repository: http://epubs.surrey.ac.uk.

Quantitative analysis of the structures in SEM images (particle spacing and quality factor for ordering); SEM and RBS analysis of normally and slowly dried hybrid films; description of Langevin dynamics simulations of structure formation; structure and optical extinction of hybrids with three different polymer particle sizes; FDTD simulations of the optical properties of hybrids; diagram showing the experimental setup for NIR radiative heating. (PDF) Movie file (AVI)

\section{AUTHOR INFORMATION}

\section{Corresponding Author}

*E-mail: j.keddie@surrey.ac.uk. 


\section{Notes}

The authors declare no competing financial interest.

\section{ACKNOWLEDGMENTS}

Funding for the Ph.D. studentship of A.U. and for a summer internship for R.T. was provided by the South East Physics Network (SEPNet). Funding for A.F. was provided by the EC BARRIER-PLUS project (number 304758 (FP7-SME-2012-2)). This work was partially supported by the University of Surrey's FRSF, Santander and IAA awards to M.F., the EPSRC (United Kingdom) DTG Grant No. EP/K503186/1, and EPSRC (United Kingdom) Strategic Equipment Grant No. EP/ M008576/1. We thank Mrs. Violeta Doukova and Mr. Chris Burt (University of Surrey) for technical assistance and Martin Murray and Phil Beharrell (Akzo Nobel, Slough) for helpful discussions. We are grateful to Akzo Nobel for the donation of the polymer colloids.

\section{REFERENCES}

(1) Linn, N. C.; Sun, C.-H.; Jiang, P.; Jiang, B. Self-Assembled Biomimetic Antireflection Coatings. Appl. Phys. Lett. 2007, 91, 101108.

(2) Zhang, X.-T.; Sato, O.; Taguchi, M.; Einaga, Y.; Murakami, T.; Fujishima, A. Self-Cleaning Particle Coating with Antireflection Properties. Chem. Mater. 2005, 17, 696-700.

(3) Anker, J. N.; Hall, W. P.; Lyandres, O.; Shah, N. C.; Zhao, J.; Van Duyne, R. P. Biosensing with Plasmonic Nanosensors. Nat. Mater. 2008, $7,442-453$.

(4) Khanadeev, V. A.; Khlebstov, B. N.; Klimova, S. A.; Tsvetkov, M. Y.; Bagratashvili, V. N.; Sukhorukov, G. B.; Khlebtsov, N. G. Large-Scale High-Quality 2D Silica Crystals: Dip-Drawing Formation and Decoration with Gold Nanorods and Nanospheres for SERS Analysis. Nanotechnology 2015, 25, 405602.

(5) Stebe, K. J.; Lewandowski, E.; Ghosh, M. Oriented Assembly of Metamaterials. Science 2009, 325, 159-160.

(6) Atwater, H. A.; Polman, A. Plasmonics for Improved Photovoltaic Devices. Nat. Mater. 2010, 9, 205-213.

(7) Cong, H.; Yu, B.; Tang, J.; Li, Z.; Liu, X. Current Status and Future Developments in Preparation and Application of Colloidal Crystals. Chem. Soc. Rev. 2013, 42, 7774-7800.

(8) Wong, S.; Kitaev, V.; Ozin, G. A. Colloidal Crystal Films: Advances in Universality and Perfection. J. Am. Chem. Soc. 2003, 125, 1558915598.

(9) Yan, Q.; Zhou, Z.; Zhao, X. S. Inward-Growing Self-Assembly of Colloidal Crystal Films on Horizontal Substrates. Langmuir 2005, 21, 3158-3164.

(10) Chung, Y.-W.; Leu, I.-C.; Lee, J.-H.; Hon, M.-H. Influence of Humidity on the Fabrication of High-Quality Colloidal Crystals Via a Capillary-Enhanced Process. Langmuir 2006, 22, 6454-6460.

(11) Zheng, Z.; Liu, X.; Luo, Y.; Cheng, B.; Zhang, D.; Meng, Q.; Wang, Y. Pressure Controlled Self-Assembly of High Quality ThreeDimensional Colloidal Photonic Crystals. Appl. Phys. Lett. 2007, 90, 051910.

(12) Cai, Z.; Teng, J.; Yan, Q.; Zhao, X. S. Solvent Effect on the SelfAssembly of Colloidal Microspheres Via a Horizontal Deposition Method. Colloids Surf., A 2012, 402, 37-44.

(13) Han, W.; Lin, Z. Learning from "Coffee Rings": Ordered Structures Enabled by Controlled Evaporative Self-Assembly. Angew. Chem., Int. Ed. 2012, 51, 1534-1546.

(14) Vogel, N.; de Viguerie, L.; Jonas, U.; Weiss, C. K.; Landfester, K. Wafer-Scale Fabrication of Ordered Binary Colloidal Monolayers with Adjustable Stoichiometries. Adv. Funct. Mater. 2001, 21, 3064-3073.

(15) Yan, Q.; Yu, J.; Cai, Z.; Zhao, X. S. Hierarchically Structured Porous Materials: From Nanoscience to Catalysis, Separation, Optics, Energy, and Life Science; Su, B.-L.; Sanchez, C.; Yang, X., Eds.; Wiley-VCH: Weinheim, 2012.

(16) Reyes, Y.; Duda, Y. Modeling of Drying in Films of Colloidal Particles. Langmuir 2005, 21, 7057-7060.
(17) Jang, H. Y.; Lee, S.-K.; Cho, S. H.; Ahn, J.-H.; Park, S. Fabrication of Metallic Nanomesh: Pt Nano-Mesh as a Proof of Concept for Stretchable and Transparent Electrodes. Chem. Mater. 2013, 25, 35353538.

(18) Li, C.; Hong, G.; Qi, L. Nanosphere Lithography at the Gas/ Liquid Interface: A General Approach toward Free-Standing HighQuality Nanonets. Chem. Mater. 2010, 22, 476-481.

(19) Bartlett, P. N.; Birkin, P. R.; Ghanem, M. A. Electrochemical Deposition of Macroporous Platinum, Palladium and Cobalt Films Using Polystyrene Latex Sphere Templates. Chem. Commun. 2000, 17, $1671-1672$.

(20) Romanov, S. G.; Korovin, A. V.; Regensburger, A.; Peschel, U. Hybrid Colloidal Plasmonic-Photonic Crystals. Adv. Mater. 2011, 23, 2515-2533.

(21) Boriskina, S. V.; Povinelli, M.; Astratov, V. N.; Zayats, A. V.; Podolskiy, V. A. Collective Phenomena in Photonic, Plasmonic and Hybrid structures. Opt. Express 2011, 19, 22024-22028.

(22) Utgenannt, A.; Keddie, J. L.; Muskens, O. L.; Kanaras, A. G. Directed Organization of Gold Nanoparticles in Polymer Coatings through Infrared-Assisted Evaporative Lithography. Chem. Commun. 2013, 49, 4253-4255.

(23) Scheeler, S. P.; Mühlig, S.; Rockstuhl, C.; Bin Hasan, S.; Ullrich, S.; Neubrech, F.; Kudera, S.; Pacholski, C. Plasmon Coupling in SelfAssembled Gold Nanoparticle-Based Honeycomb Islands. J. Phys. Chem. C 2013, 117, 18634-18641.

(24) Cintra, S.; Abdelsalam, M. E.; Bartlett, P. N.; Baumberg, J. J.; Kelf, T. A.; Sugawara, Y.; Russell, A. E. Sculpted Substrates for SERS. Faraday Discuss. 2006, 132, 191-199.

(25) Routh, A. F.; Zimmerman, W. B. Distribution of Particles during Solvent Evaporation from Films. Chem. Eng. Sci. 2004, 59, 2961-2968.

(26) Trueman, R. E.; Lago Domingues, E.; Emmett, S. N.; Murray, M. W.; Routh, A. F. Auto-Stratification in Drying Colloidal Dispersions: A Diffusive Model. J. Colloid Interface Sci. 2012, 377, 207-212.

(27) Cardinal, C. M.; Jung, Y. D.; Ahn, K. H.; Francis, L. F. Drying Regime Maps for Particulate Coatings. AIChE J. 2010, 56, 2769-2780.

(28) Romanov, S. G.; Susha, A. S.; Sotomayor Torres, C. M.; Liang, Z.; Caruso, F. Surface Plasmon Resonance in Gold Nanoparticle Infiltrated Dielectric Opals. J. Appl. Phys. 2005, 97, 086103.

(29) Morandi, V.; Marabelli, F.; Amendola, V.; Meneghetti, M.; Comoretto, D. Colloidal Photonic Crystals Doped with Gold Nanoparticles: Spectroscopy and Optical Switching Properties. Adv. Funct. Mater. 2007, 17, 2779-2786.

(30) Wang, D.; Li, J.; Chan, C. T.; Salgueirico-Maceira, V.; Liz-Marzan, L. M.; Romanov, S.; Caruso, F. Optical Properties of NanoparticleBased Metallodielectric Inverse Opals. Small 2005, 1, 122-130.

(31) Halas, N. J.; Lal, S.; Chang, W.-S.; Link, S.; Nordlander, P. Plasmons in Strongly Coupled Metallic Nanostructures. Chem. Rev. 2011, 111, 3913-3961.

(32) Zhong, Z.; Patskovskyy, S.; Bouvrette, P.; Luong, J. H. T.; Gedanken, A. The Surface Chemistry of Au Colloids and Their Interactions with Functional Amino Acids. J. Phys. Chem. B 2004, 108, 4046-4052.

(33) Barrow, S. J.; Wei, X.; Baldauf, J. S.; Funston, A. M.; Mulvaney, P. The Surface Plasmon Modes of Self-Assembled Gold Nanocrystals. Nat. Commun. 2012, 3, 1275.

(34) Kelf, T. A.; Sugawara, Y.; Cole, R. M.; Baumberg, J.J.; Abdelsalam, M. E.; Cintra, S.; Mahajan, S.; Russell, A. E.; Bartlett, P. N. Localized and Delocalized Plasmons in Metallic Nanovoids. Phys. Rev. B: Condens. Matter Mater. Phys. 2006, 74, 245415.

(35) Lin, T.-L.; Lin, J.-H.; Guo, J.-T.; Kan, H.-C. Suppression of Photonic Bandgap Reflection by Localized Surface Plasmons in SelfAssembled Plasmonic-Photonic Crystals. Adv. Opt. Mater. 2015, 3, $1470-1475$

(36) Robbiano, V.; Giordano, M.; Martella, C.; Di Stasio, F.; Chiappe, D.; Buatier de Mongeot, F.; Comoretto, D. Hybrid PlasmonicPhotonic Nanostructures: Gold Nanocrescents Over Opals. Adv. Opt. Mater. 2013, 1, 389-396.

(37) Morandi, V.; Marabelli, F.; Amendola, V.; Meneghetti, M.; Comoretto, D. Light Localization Effect on the Optical Properties of 
Opals Doped with Gold Nanoparticles. J. Phys. Chem. C 2008, 112, 6293-6298.

(38) Bahns, J. T.; Guo, Q.; Montgomery, J. M.; Gray, S. K.; Jaeger, H. M.; Chen, L. High-Fidelity Nano-Hole-Enhanced Raman Spectroscopy. J. Phys. Chem. C 2009, 113, 11190-11197.

(39) Sánchez-Sobrado, O.; Lozano, G.; Calvo, M. E.; Sánchez-Iglesias, A.; Liz-Marzán, L. M.; Míguez, H. Interplay of Resonant Cavity Modes with Localized Surface Plasmons: Optical Absorption Properties of Bragg Stacks Integrating Gold Nanoparticles. Adv. Mater. 2011, 23, 2108-2112.

(40) Yoo, S.; Park, Q.-H. Effective Permittivity for Resonant Plasmonic Nanoparticle Systems Via Dressed Polarizability. Opt. Express 2012, 20, 16480-16489.

(41) Turkevich, J.; Stevenson, P. C.; Hillier, J. A Study of the Nucleation and Growth Process in the Synthesis of Colloidal Gold. Discuss. Faraday Soc. 1951, 11, 55-75.

(42) Loweth, C. J.; Caldwell, W. B.; Peng, X.; Alivisatos, A. P.; Schultz, P. G. DNA-Based Assembly of Gold Nanocrystals. Angew. Chem., Int. Ed. 1999, 5, 1808-1812.

(43) Haiss, W.; Thanh, N. T. K.; Aveyard, J.; Fernig, D. G. Determination of Size and Concentration of Gold Nanoparticles from UV-Vis Spectra. Anal. Chem. 2007, 79, 4215-4221.

(44) Georgiadis, A.; Bryant, P. A.; Murray, M.; Beharrell, P.; Keddie, J. L. Resolving the Film Formation Dilemma with Infrared RadiationAssisted Sintering. Langmuir 2011, 27, 2176-2180.

(45) Simon, A.; Jeynes, C.; Webb, R. P.; Finnis, R.; Tabatabaian, Z.; Sellin, P. J.; Breese, M. B. H.; Fellows, D. F.; van den Broek, R; Gwilliam, R. M. The New Surrey Ion Beam Analysis Facility. Nucl. Instrum. Methods Phys. Res., Sect. B 2004, 219-220, 405-409.

(46) Jeynes, C.; Bailey, M. J.; Bright, N. J.; Christopher, M. E.; Grime, G. W.; Jones, B. N.; Palitsin, V. V.; Webb, R. P. Total IBA" - Where Are We? Nucl. Instrum. Methods Phys. Res., Sect. B 2012, 271, 107-118.

(47) Barradas, N. P.; Jeynes, C. Advanced Physics and Algorithms in the IBA DataFurnace. Nucl. Instrum. Methods Phys. Res., Sect. B 2008, 266, 1875-1879.

(48) Plimpton, S. Fast Parallel Algorithms for Short-Range Molecular Dynamics. J. Comput. Phys. 1995, 117, 1-19. 